\title{
Pubertal and testicular development in the common marmoset (Callithrix jacchus) shows high individual variation
}

\author{
S. Irfan ${ }^{1,2}$, J. Wistuba ${ }^{1}$, J. Ehmcke $^{1}$, M. Shahab ${ }^{2}$, and S. Schlatt ${ }^{1}$ \\ ${ }^{1}$ Institute of Reproductive and Regenerative Biology, Center of Reproductive Medicine and Andrology, \\ University Clinics, Münster, Germany \\ ${ }^{2}$ Department of Animal Sciences, Faculty of Biological Sciences, Quaid-i-Azam University, Islamabad, \\ Pakistan
}

Correspondence to: S. Schlatt (stefan.schlatt@ukmuenster.de)

Received: 22 December 2014 - Revised: 12 March 2015 - Accepted: 23 March 2015 - Published: 13 April 2015

\begin{abstract}
The common marmoset (Callithrix jacchus) is a New World primate that exhibits a man-like adult testicular organization. Aims: this study examines the pubertal testicular development in the common marmoset. Material and methods: immature male common marmosets $(n=48)$ were monitored longitudinally for a period of 13 months. Body weight and testicular volume (TV) were recorded, and testosterone levels were analyzed by an in-house radioimmunoassay. After 13 months the testes were collected, fixed and embedded in paraffin $(n=48)$. Histological and morphometric data were determined. Results: the first 6 months exhibited a rapid rise in body weight but not in TV. At 7 months a threefold increase in testosterone levels was observed. After 7 months the first few animals displayed rapid testis growth $\left(>250 \mathrm{~mm}^{3}\right.$ at 10 months), while others exhibited no or slow pubertal development ( $\leq 100 \mathrm{~mm}^{3}$ at 10 months). Histological features confirmed an individually variable pattern of testicular development. Parallel with the rise in serum testosterone levels, an increase in the diameter of seminiferous tubules and an appearance of a tubular lumen as well as meiotic germ cells were encountered. The onset and the kinetics of testicular development were highly variable between individual animals in the colony. Epididymal sperm were first observed at 12 months of age. The TV and seminiferous tubule diameter showed continued growth after 12 months of age, especially in the animals developing with a delay after 7 months. Conclusions: pubertal onset in the common marmosets occurs at the earliest at 6 months of age and is hallmarked by sudden threefold increase in serum testosterone levels and a significant rise in the TV. Pubertal testis growth is characterized by an appearance of a tubular lumen and of primary and secondary spermatocytes. Spermatogenesis is qualitatively accomplished at the earliest at 12 months of age. A very high individual difference in onset and kinetics of pubertal development renders the age a very poor prognostic factor to determine the pubertal status of individual marmosets.
\end{abstract}

\section{Introduction}

The common marmoset (Callithrix jacchus) is considered a valuable laboratory animal in biomedical research and toxicology owing to its small size, high breeding rate and longer life span in captivity (Abbott et al., 2003; Mansfield, 2003; Zühlke and Weinbauer, 2003; 't Hart et al., 2012). Seminiferous tubular organization in adult male common marmosets shows high similarity to human testis. The fetal and neonatal male germ cell development in the common marmoset mimics human pattern of pre- and postnatal development (Mitchell et al., 2008; Millar et al., 2000; Wistuba et al., 2003). These significant observations highlight common marmoset as a valid non-human primate model for the study of primate specific features in testicular germ cell development (Gassei and Schlatt, 2007).

In primarily descriptive publications the pubertal onset in the common marmoset has been characterized by serum 
testosterone levels and a rise in testis volume (TV) during postnatal development (Abbot and Hearn, 1978; Jackson and Edmonds, 1984). Experimental manipulations were adopted to unravel the postnatal aspect of testicular development in immature common marmosets (Lunn et al., 1994, 1997; Sharpe et al., 2000, 2003a, b). Some features in marmosets are specific for New World monkeys. For example the pituitary gland in the common marmoset releases a chorionic gonadotropin (CG)-like molecule, having a much shorter serum half-life as compared with Luteinizing Hormone (LH; Muller et al., 2004). In the common marmoset the pubertal reactivation of hypothalamic pituitary gonadal axis, characterized by elevated levels of testosterone, is currently considered to occur between 6 and 12 months of age (Li et al., 2005). Keeping in mind aspects of the unique reproductive endocrinology of common marmoset, we have previously documented the pubertal development in the common marmoset employing a cross-sectional approach (Chandolia et al., 2006).

Here we aimed to investigate the pubertal growth in the common marmoset by observing immature common marmosets longitudinally for a period of 13 months. Our primary aims included (1) exploring the precise timing of pubertal onset in terms of hypothalamic pituitary gonadal axis activation and (2) characterizing the morphological features in the testis before, during and after pubertal onset. Our secondary aims were to correlate the somatic and reproductive growth pattern and to measure the time required by the immature testis to fully establish spermatogenesis qualitatively.

\section{Materials and methods}

\subsection{Animals and study design}

Immature common marmosets were observed for 13 months starting either from birth or at an age of less than 12 months. Animals were housed in the animal facility of the University Hospital Münster (UKM). Animals were either housed with their families or were separated into groups of the same sex and age ( \pm 1 month). Animals were fed a complex diet consisting of commercially available powdered baby and canned cat food, fresh fruits, vegetables, mealworms, marmoset pellets and vitamin supplements. Water was available from bottles ad libitum.

The study was designed to longitudinally monitor animals for body weight, testicular volume and serum testosterone during the postnatal development. In order to document the testicular histomorphometric changes from neonatal phase to post-pubertal phase, groups of monkeys were sacrificed from birth to 12 months of age and during adulthood. Table 1 demonstrates the different age groups and the number of animals present in each age group.

\subsection{Sampling}

Monthly body weight (balance) and testicular volume (caliper) were recorded under manual restraint. At the same time blood samples were collected from the femoral vein of the animals. The samples were collected between 08.00 and 10.30 a.m. The serum was separated from the blood samples and stored at $-20^{\circ} \mathrm{C}$ till assayed. Testis length and width were measured using vernier caliper, and the testicular volume was calculated using an established ellipsoid formula $\left(\mathrm{TV}=\left(W^{2} \cdot L\right) \cdot 3.141592654 / 6\right)$.

\subsection{Hormone assay}

Testosterone (T) was assayed in serum samples using an in-house radioimmunoassay (RIA) method. Serum testosterone was measured by a solid-phase, double-antibody RIA technique, using a commercially available iodinated tracer (testosterone-3-(0-carboxymethyl) oximino2-[12T] iodohistamine; Amersham International, Braunschweig, Germany) and an antiserum raised in rabbits against testosterone-3(carboxymethyloxime)-BSA. The bound/free separation was performed by addition of a solution of solidphase antirabbit immunoglobulins (Immunobead Second Antibody, Biorad, Munich, Germany). The recovery after ether extraction was monitored by addition of trace amounts of $[1 \beta, 2 \beta-3 \mathrm{H}]$ testosterone (NET-187, NEN, Boston, MA), and the final results were corrected accordingly. The sensitivity was $2 \mathrm{pg}$ tube $^{-1}(0.07 \mathrm{nmol} / 1)$. In 10 consecutive assays the intra-assay coefficients of variation (mean \pm SEM) were $8.43+1.42,4.2+0.59$ and $4.37+0.63 \%$ for control sera with low, middle and high testosterone concentrations, respectively. The corresponding inter-assay coefficients of variations were $16.62,6.26$ and $3.85 \%$, respectively.

\subsection{Tissue collection and processing}

At the end of the study each animal was deeply anesthetized with ketamine, and body weight and testicular volume were recorded. Subsequently the animals were sacrificed by exsanguination. The weight of testis tissues were recorded immediately after removal. Testis tissue was fixed in Bouin's solution overnight and later routinely embedded in paraffin. Sections of $3 \mu \mathrm{m}$ thickness were cut. A total of six sections from six separate regions of the tissue were taken from the testis of the each animal. Periodic acid-Schiff staining was performed on these sections.

\subsection{Histological recordings}

Slides were analyzed using an Olympus BX61 microscope (Melville, NY, USA) with an attached Retiga 4000R camera (QImaging, Surrey, BC, Canada). All images were acquired digitally using QCapture imaging software (QImaging, Surrey, BC, Canada). Pictures were taken from five independent 


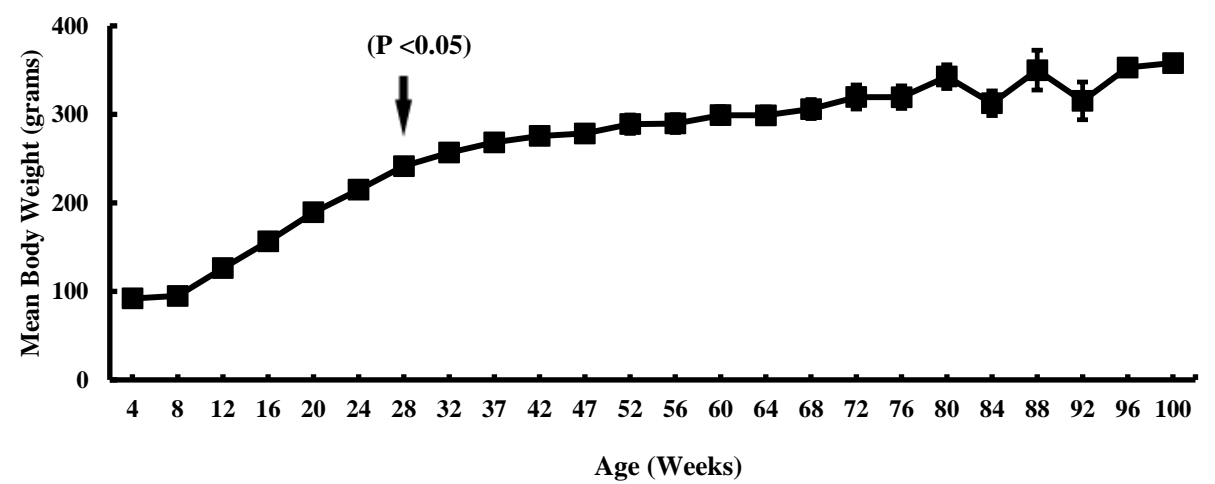

Figure 1. Mean body weight in the common marmoset from birth until 100 weeks of age $(n=48)$. In the first 28 weeks a significant $(P<0.05)$ (arrow) increase in the body weight was observed as compared with mean values at 24, 20 and 16 weeks of age.

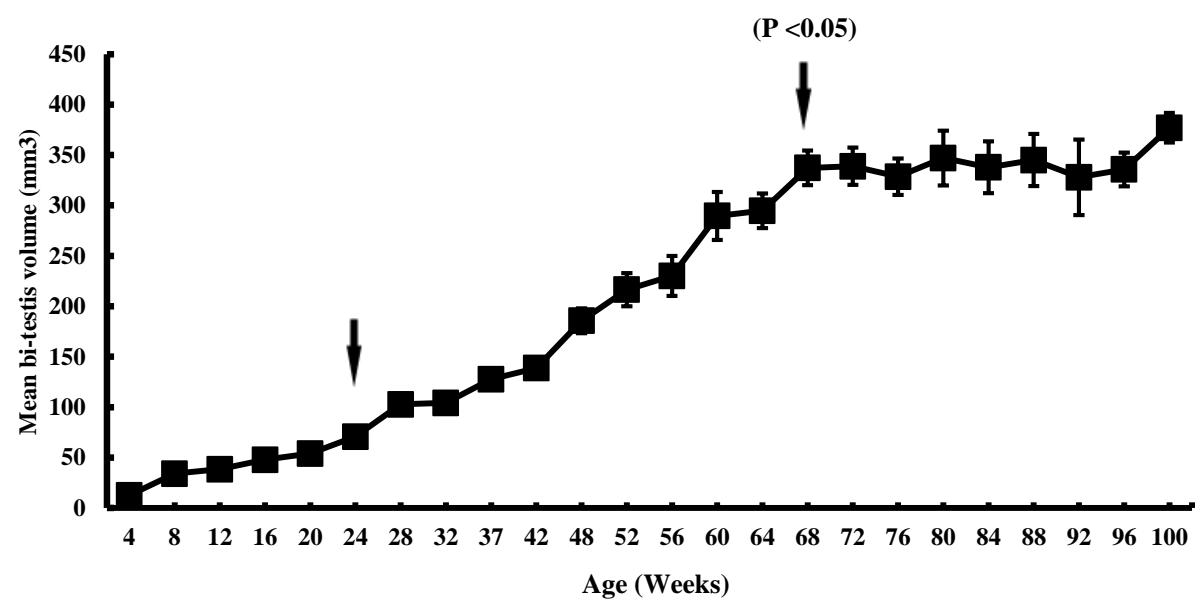

Figure 2. Mean bi-testis volume in the common marmoset from birth until 100 weeks of age $(n=48)$. A rapid and statistically significant $(P<0.05)$ increase in the testis volume was observed from the 24 th week onwards until the 68 th week of age (arrow). This rapid increase in the testis volume was absent from the 68th week onwards.

positions from each section at $20 \times$ (five pictures per section) using the camera fitted on the microscope. A total of 30 pictures from each testis were used for point counting and morphometric measurements.

\subsection{Histomorphometric procedures}

Details of the morphometry have been described previously (Schlatt et al., 1999). Volume density expressed as the relative proportion of testicular tissue was determined by point counting using a random systemic and blinded approach on six sections from each testis. A total of 120 points were scored for each testis. An ocular grid showing a square was used, and the four corners of the grid were analyzed to be located on interstitium, tubule epithelium or tubule lumen. Volume density of various components multiplied by testis weight (assuming a specific weight of testis tissue of 1) yielded the total weight of that component per testis. Sixty measurements were taken from each testis (10 mea- surements from each section). Round tubules were selected for absolute measurement of the tubule diameter.

\subsection{Statistics}

All data were expressed as mean \pm SEM unless stated otherwise. Student's $t$ test was employed to analyze the statistical significance. $P$ value was set at 0.05 for the results to be considered as significant.

\section{Results}

\subsection{Mean body weight, mean bi-testis volume and mean plasma testosterone}

The mean body weight in immature common marmosets showed robust growth during the first 28 weeks (7 months) of age. The mean body weight at 7 months was significantly $(P<0.05)$ higher as compared to the mean body weight at 4, 5 and 6 months of age. From then onwards no statistically 


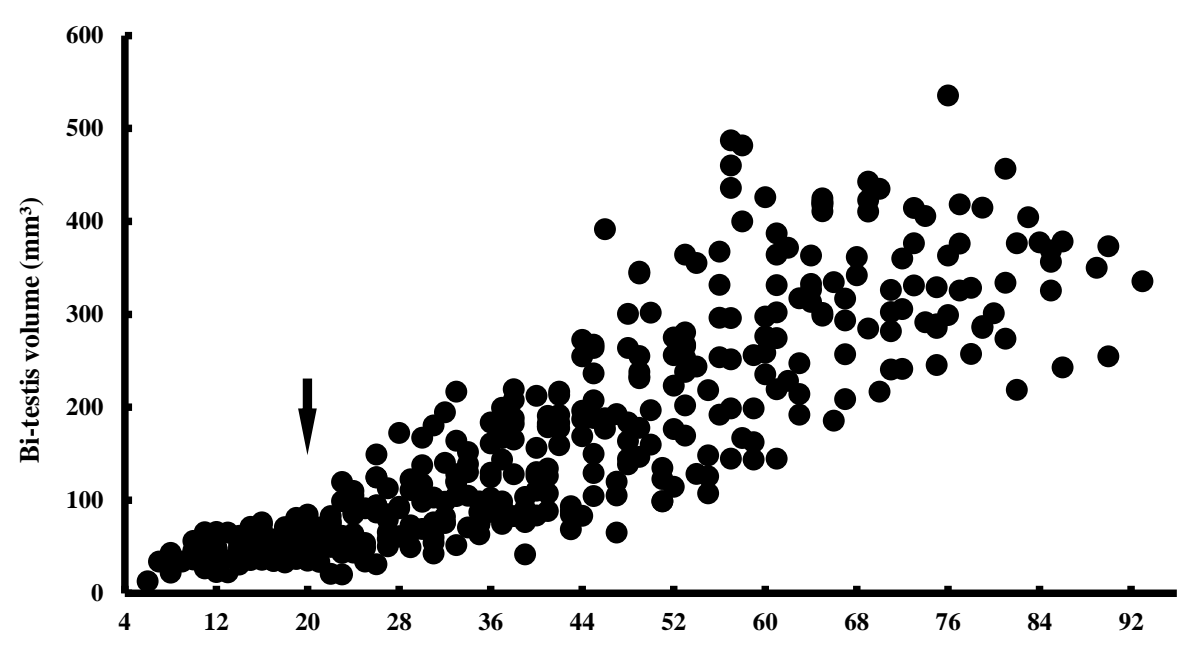

Age (weeks)

Figure 3. Bi-testis volume of the individual common marmosets $(n=48)$. Note the homogenous testis volume until the 20 th week $(5$ months $)$ of age (arrow), while highly variable individual testicular volume was observed after the 24 th week (6 months) of age.

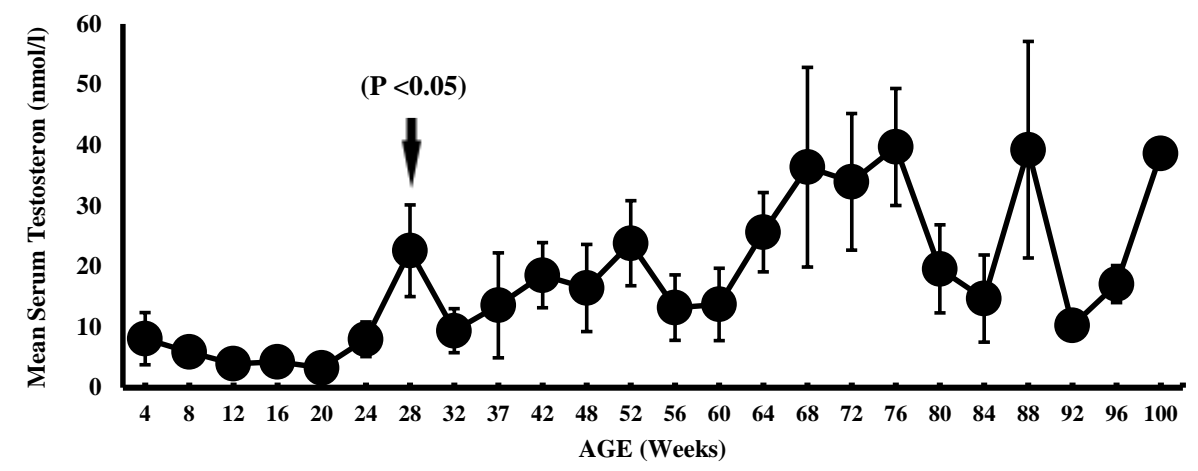

Figure 4. Mean serum testosterone levels from birth until the 100th week of age in the common marmoset $(n=48)$. Note the first significant $(P<0.05)$ (arrow) rise in the mean serum testosterone at the 28 th week of age as compared to 24,20 and 16 weeks of age, followed by continuously high serum testosterone levels observed afterwards.

significant increase in body weight was observed (Fig. 1). The mean bi-testis volume shows no statistically significant increase until the 24th week (6 months) of age, followed by a statistically significant $(P<0.05)$ increase until 68 weeks (16 months) of age in comparison with the mean bi-testis volume at 24 weeks of age (Fig. 2). While the testis volumes of the individual animals were homologically low until 20 weeks (Fig. 3), a high individual variability in testis volume was observed thereafter. An individually diverse pattern was recorded, with some animals reaching adult testis size $\left(>250 \mathrm{~mm}^{3}\right)$ at 44 weeks (9-10 months) while other show slow or no increase in the testis volume $\left(\leq 100 \mathrm{~mm}^{3}\right)$ until 10 months of age. Interestingly animals showing an early or late onset of pubertal development show comparable body weights and testicular histological features (Fig. 1, Table 1).

Mean serum testosterone levels depicted a sudden and significant $(P<0.05)$ rise in serum testosterone levels at the 28th week (7 months) of age (Fig. 4). The threefold rise in serum testosterone at this time point was the first endocrine sign of pubertal activation of the hypothalamic pituitary gonadal axis and correlated with the first increase of testicular volume. No prepubertal significant rise in testosterone levels were observed earlier than 7 months of age. However the mean serum testosterone levels were slightly higher $\left(8.5 \mathrm{nmol} \mathrm{L}^{-1}\right)$ in the first month of the study period compared to the following months.

Testis weights were determined in all 48 monkeys at the time of sacrifice. These values are relevant for comparison with the histological analysis. The absolute testis weights and volumes as well as relative testis weights in relation to body weight are shown in Figs. 8 and 9. 
Table 1. Description of advanced germ cell type, Sertoli cell arrangement and the presence or absence of sperm in the epididymis of common marmosets over the course of development $(n=48)$.

\begin{tabular}{|c|c|c|c|}
\hline Age & Most advanced germ cell type & Sertoli cell nuclei arrangement & Epididymal sperm \\
\hline 4 months, $n=6$ & Gonocytes & Random arrangement & No \\
\hline 5 months, $n=3$ & A spermatogonia & Random arrangement & No \\
\hline 6 months, $n=6$ & A spermatogonia & Epithelial arrangement & No \\
\hline 7 months, $n=3$ & $\begin{array}{l}\text { B spermatogonia, } \\
\text { few primary spermatocytes }\end{array}$ & Epithelial arrangement & No \\
\hline 8 months, $n=5$ & Round spermatids & Epithelial arrangement & No \\
\hline 10 months, $n=3$ & $\begin{array}{l}\text { Round spermatids, } \\
\text { few elongating spermatids }\end{array}$ & Epithelial arrangement & No \\
\hline $12-20$ months $n=22$ & Elongating spermatids & Epithelial arrangement & Yes \\
\hline
\end{tabular}

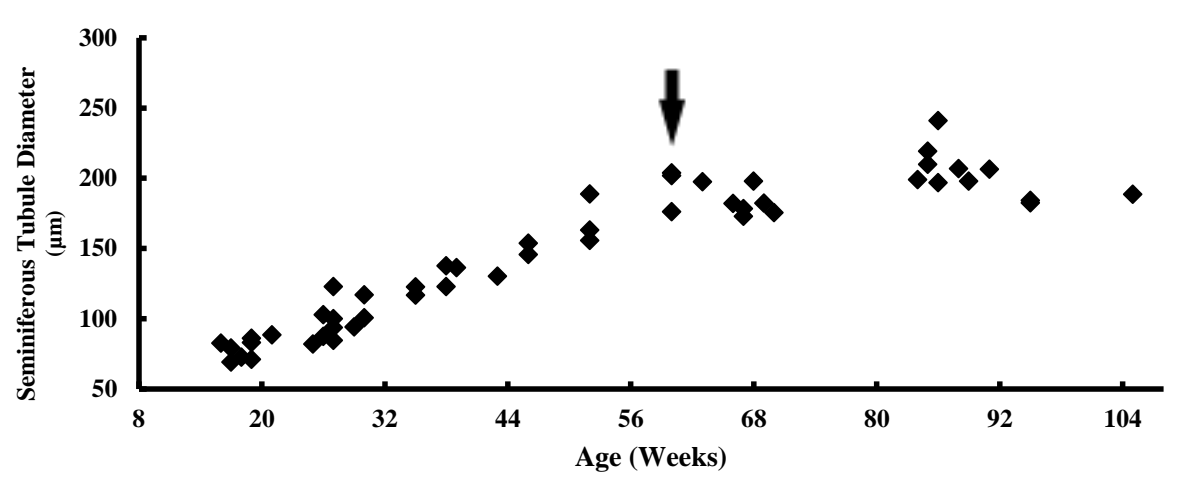

Figure 5. Mean individual seminiferous tubule diameter from 16 weeks onwards till 104 weeks in individual common marmosets $(n=48)$. Increments in the mean tubule diameter until 60 weeks of age were statistically significant $(P<0.05)$ as compared to the mean tubule diameter at 24 weeks of age.

\subsection{Testicular histomorphometric analysis}

The seminiferous tubule diameter increased initially at 24 weeks and continued to rise until 68 weeks of age (Fig. 5). The presence of a lumen was first observed at 24 weeks (Figs. 6 and 7). With advancing age the relative proportions of the volume densities of the various compartments remain relatively constant; however, when expressed as absolute weights of each compartment the significant growth of the tubular compartment during population with differentiating germ cells is obvious (Fig. 7). Until 20 weeks Sertoli cell nuclei were randomly distributed in the cords. At 6 months of age the Sertoli cells are arranged towards the periphery of the seminiferous tubules in an epithelial-like fashion (Table 1). Gonocytes were no longer observed after 5 months of age, and A spermatogonia were present from around 6 months of age. B spermatogonia and few primary spermatocytes were first observed around 7 months of age, and round spermatids were first observed at 8 months of age, whereas sperm were first detected in the epididymis at 12 months of age (Table 1).

\section{Discussion}

Testicular pubertal development in the common marmoset was examined in the present study. No experimental manipulation was performed in this study, and all data were obtained by recordings of animals in family breeding or samesex group holding conditions. We assume that these conditions provide the environmental conditions for normal postnatal growth patterns in laboratory colonies. The primary aim of the present study was the identification of the initial age of pubertal onset, which was monitored by testis volume and serum testosterone levels. We observed that the initial pubertal activation of the hypothalamic pituitary gonadal axis in terms of high serum levels of testosterone in the common marmoset occurs after 6 months ( 24 weeks). This finding is in agreement with the observation by Abbot and Hearn (1978). We therefore see a slightly earlier initiation of puberty than reported by Lunn et al. (1994) and Kelnar et al. (2002).

First seminiferous lumen formation was observed after 6 months at a time when high serum testosterone was first encountered. It can be speculated that an expression of androgen receptors by Sertoli cells during this developmental period window is causing the onset of Sertoli cell secretory 


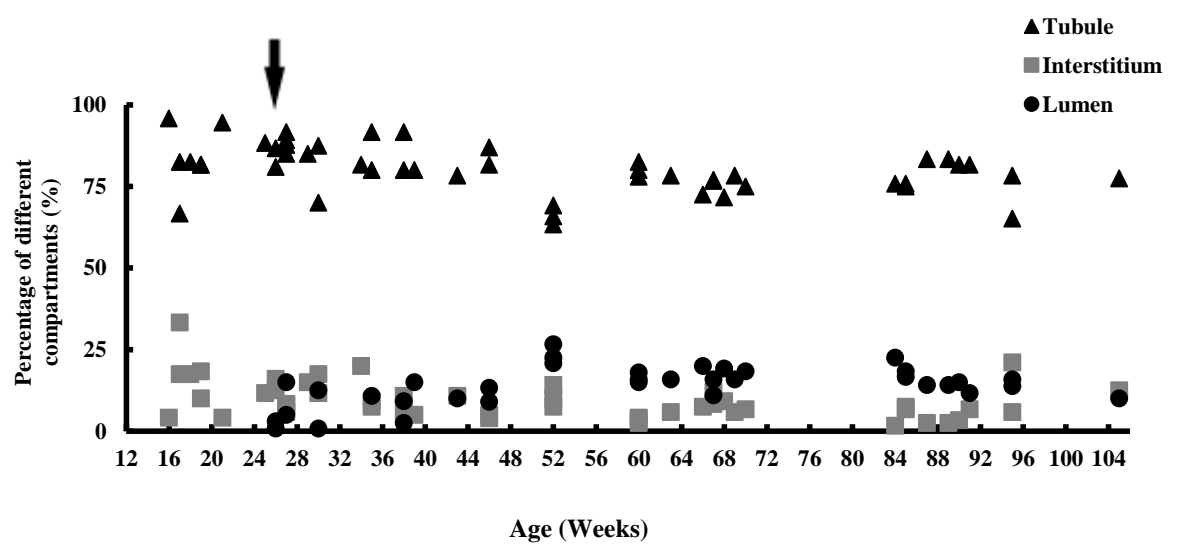

Figure 6. Percentage of seminiferous tubules, interstitial area and tubule lumen during development in individual common marmosets $(n=48)$. The high percentage of the interstitial compartment until 20 weeks ( 5 months) of age is followed by the presence of lumen after 24 weeks (6 months) of age (arrow).

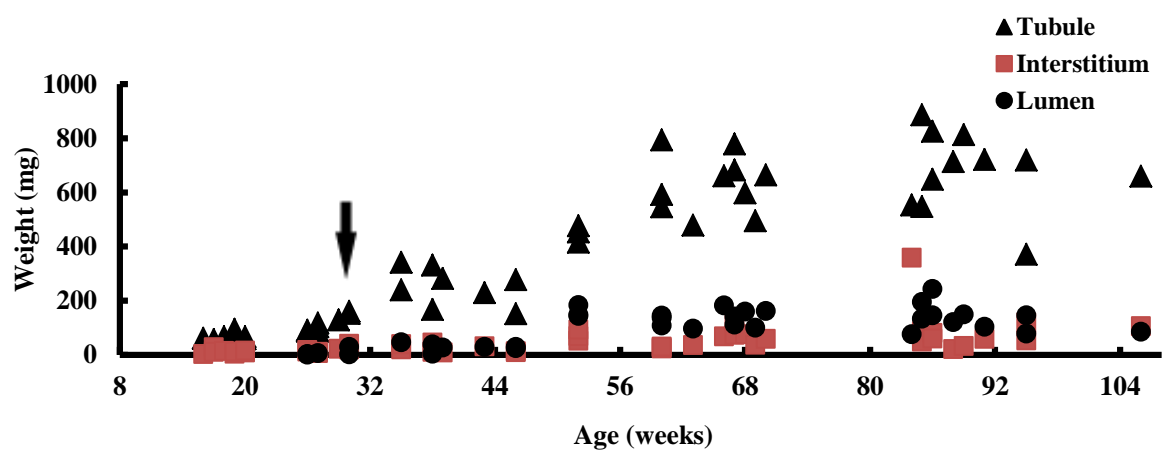

Figure 7. Calculated weight of seminiferous epithelium, interstitial area and the tubule lumen during development in the individual common marmosets $(n=48)$. The rapid increase in the tubular weight was observed after 30 weeks of age (arrow).

activity and establishment of the blood testis barrier by Sertoli cell tight and gap junctions, which will be recognized through the formation of seminiferous lumen and diametrical tubule growth (Majumdar et al., 2012). The presence of postmeiotic germ cells around 10 months of age indicated that at this time the blood testis barrier has been established by adjacent Sertoli cells (Dym, 1972). We observed a significant, individually highly variable increase in the testicular volume between 6 and 16 months of age. The histological analysis of the testis tissue revealed that the qualitative completion of spermatogenesis was not achieved prior to 12 months. The increase in the testis volume which was observed after 12 months of age seems to be attributed only to expansion of the differentiating germ cell population. Jackson and Edmonds (1984) pointed out that the 60th week (14 months) of age can be considered as the age when testicular maturity in terms of full establishment of spermatogenesis occurs. In our study a surprisingly variable development was observed. In terms of kinetics the most rapid development required to establish qualitatively full spermatogenesis from the first sign of puberty until maximal growth of the testis was 5 months. However many monkeys did show a very significant delay in the onset of puberty or a much longer period to go through the pubertal process, revealing an individually highly diverse picture of pubertal animals in the colony for which age is a poor predictor. Between 40 and 60 weeks an individual monkey could also be at a very early stage of pubertal development or already showing full testis size with qualitatively normal spermatogenesis. Chandolia et al. (2006) have previously documented the pubertal development in the common marmoset using a cross-sectional study design. As onset and kinetics of primate puberty seem to be variable, the cross-sectional study design has its limitations in observing all details of the pubertal process. The exact timing of the pubertal onset in terms of serum testosterone and testis growth can best be observed by a longitudinal study design. In addition individual variations between animals cannot be characterized in cross-sectional study design. In the present study we documented not only the timing of the pubertal onset but also the time period required to accomplish pubertal maturation until completion of spermatogenesis after initiation of puberty.

Our data concerning the body weight gain depicted an important aspect of postnatal development in the common mar- 

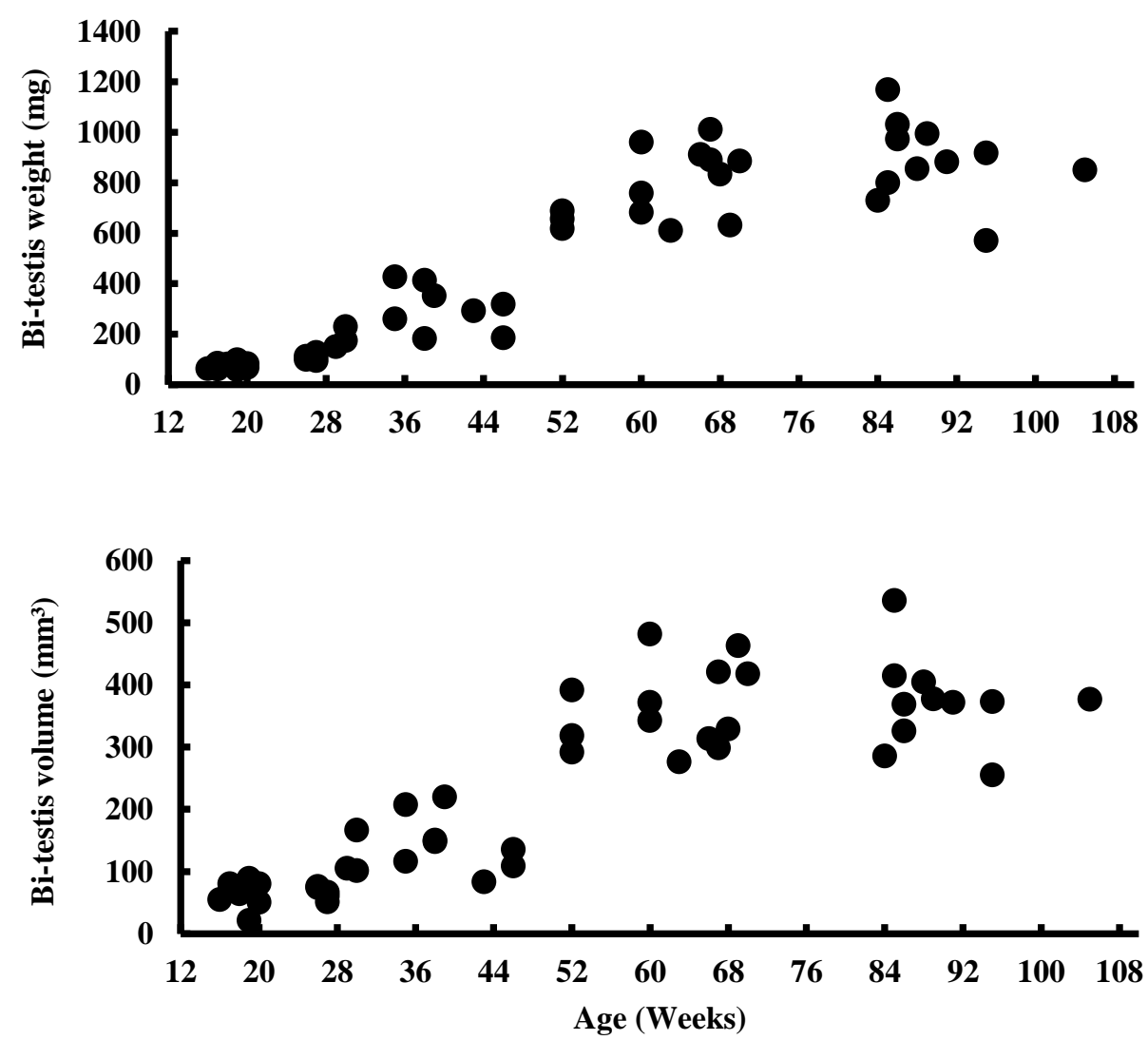

Figure 8. Bi-testis weight and bi-testis volume of the sacrificed individual common marmosets $(n=48)$.

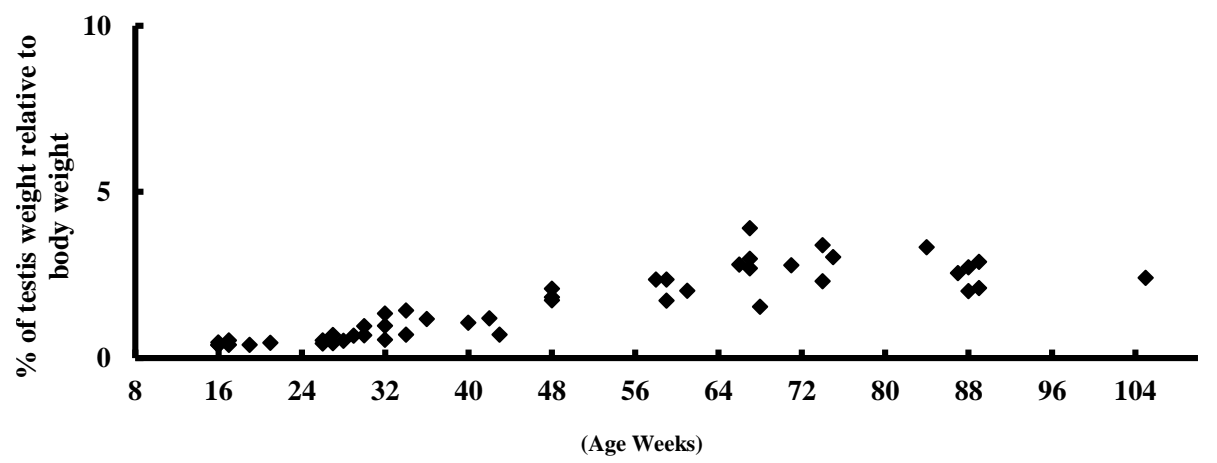

Figure 9. Relative percentage of the bi-testis weight to the body weight in the sacrificed animals $(n=48)$.

moset. The maximum body weight gain had been observed well within the first 6 months of age before the pubertal activation. After 6 months the animals showed a slower rise in the body weight. Whether the initial weight gain is a prerequisite for the pubertal activation remains to be explored. It seems that growth and sexual development are controlled independently in the common marmoset.

\section{Conclusions}

In conclusion pubertal testicular development in the common marmoset initiates at 6 months of age at the earliest and is visible by a parallel rise in testosterone and testicular growth. The pubertal onset in the common marmoset is preceded by a period of accelerated somatic growth. Qualitative completion of spermatogenesis occurs at 12 months of age at the earliest. The actual start and the kinetics of puberty changes are individually highly variable. 
Acknowledgements. The work presented here was supported by a DAAD fellowship grant to Shahzad Irfan and by a DFG project (FOR 1041, project 3). We thank Martin Heuermann and Günter Stelke for excellent care and management of the marmoset breeding colony. We also like to thank Reinhild SandhoweKlaverkamp for skilled technical assistance with the testosterone radioimmunoassay and Jutta Salzig for support on histological procedures.

Edited by: E. Fuchs

Reviewed by: two anonymous referees

\section{References}

Abbott, D. H., Barnett, D. K., Colman, R. J., Yamamoto, M. E., and Schultz-Darken, N. J.: Aspects of common marmoset basic biology and life history important for biomedical research, Comp. Med., 53, 339-350, 2003.

Abbott, D. H. and Hearn, J. P.: Physical, hormonal and behavioral aspects of sexual development in the marmoset monkey, Callithrix jacchus, J. Reprod. Fertil., 53, 155-166, 1978.

Chandolia, R. K., Luetjens, C. M., Wistuba, J., Yeung, C. H., Nieschlag, E., and Simoni, M.: Changes in endocrine profile and reproductive organs during puberty in the male marmoset monkey (Callithrix jacchus), Reproduction, 132, 355-363, 2006.

Dym, M.: The fine structure of the monkey (Macaca mulatta) Sertoli cell and its role in maintaining the blood-testis barrier, Anat. Rec., 175, 639-656, 1972.

Gassei, K. and Schlatt, S.: Testicular morphogenesis: comparison of in vivo and in vitro models to study male gonadal development, Ann. NY Acad. Sci., 1120, 152-167, 2007.

Jackson, M. R. and Edmunds, J. G.: Morphological assessment of testicular maturity in marmosets (Callithrix jacchus), Lab. Anim., 18, 173-178, 1984.

Kelnar, C. J. H., McKinnell, C., Walker, M., Morris, K. D., Wallace, W. H. B., Saunders, P. T. K., Fraser, H. M., and Sharpe, R. M.: Testicular changes during infantile "quiescence" in the marmoset and their gonadotrophin dependence: a model for investigating susceptibility of the prepubertal human testis to cancer therapy?, Hum. Reprod., 17, 1367-1378, 2002.

Li, L. H., Donald, J. M., and Golub, M. S.: Review on testicular development, structure, function, and regulation in common marmoset, Birth. Defects Res. B, 74, 450-469, 2005.

Lunn, S. F., Recio, R., Morris, K., and Fraser, H. M.: Blockade of the neonatal rise in testosterone by a gonadotrophin-releasing hormone antagonist: effects on timing of puberty and sexual behaviour in the male marmoset monkey, J. Endocrinol., 141, 439447, 1994.

Lunn, S. F., Cowen, G. M., and Fraser, H. M.: Blockade of the neonatal increase in testosterone by a GnRH antagonist: the free androgen index, reproductive capacity and postmortem findings in the male marmoset monkey, J. Endocrinol., 154, 125-131, 1997.
Mansfield, K.: Marmoset models commonly used in biomedical research, Comp. Med., 53, 383-392, 2003.

Majumdar, S. S., Sarda, K., Bhattacharya, I., and Plant, T. M.: Insufficient androgen and FSH signaling may be responsible for the azoospermia of the infantile primate testes despite exposure to an adult-like hormonal milieu, Hum. Reprod., 27, 2515-2525, 2012.

Millar, M. R., Sharpe, R. M., Weinbauer, G. F., Fraser, H. M., and Saunders, P. T.: Marmoset spermatogenesis: organizational similarities to the human, Int. J. Androl., 23, 266-277, 2000.

Mitchell, R. T., Cowan, G., Morris, K. D., Anderson, R. A., Fraser, H. M., Mckenzie, K. J., Wallace, W. H., Kelnar, C. J., Saunders, P. T., and Sharpe, R. M.: Germ cell differentiation in the marmoset (Callithrix jacchus) during fetal and neonatal life closely parallels that in the human, Hum. Reprod. 23, 2755-2765, 2008.

Muller, T., Simoni, M., Pekel, E., Luetjens, C. M., Chandolia, R., Amato, F., Norman, R. J., and Gromoll, J.: Chorionic gonadotrophin beta subunit mRNA but not luteinising hormone beta subunit mRNA is expressed in the pituitary of the common marmoset (Callithrix jacchus), J. Mol. Endocrinol., 32, 115-128, 2004.

Schlatt, S., Zhengwei, Y., Meehan, T., de Kretser, D. M., and Loveland, K. T.: Application of morphometric techniques to postnatal rat testes in organ culture: insights into testis growth, Cell Tissue Res., 298, 335-343, 1999.

Sharpe, R. M., Walker, M., Millar, M. R., Atanassova, N., Morris, K., McKinnell, C., Saunders, P. T., and Fraser, H. M.: Effect of neonatal gonadotropin-releasing hormone antagonist administration on Sertoli cell number and testicular development in the marmoset: comparison with the rat, Biol. Reprod., 62, 1685-1693, 2000.

Sharpe, R. M., Fraser, H. M., Brougham, M. F., McKinnell, C., Morris, K. D., Kelnar, C. J., Wallace, W. H., and Walker, M.: Role of the neonatal period of pituitary-testicular activity in germ cell proliferation and differentiation in the primate testis, Hum. Reprod., 18, 2110-2117, 2003a.

Sharpe, R. M., McKinnell, C., Kivlin, C., and Fisher, J. S.: Proliferation and functional maturation of Sertoli cells, and their relevance to disorders of testis function in adulthood, Reproduction, 125, 769-784, 2003b.

't Hart, B. A., Abbott, D. H., Nakamura, K., and Fuchs, E.: The marmoset monkey: a multi-purpose preclinical and translational model of human biology and disease, Drug Discov. Today, 17, 1160-1165, 2012.

Wistuba, J., Schrod, A., Greve, B., Hodges, J. K., Aslam, H., Weinbauer, G. F., and Luetjens, C. M.: Organization of seminiferous epithelium in primates: relationship to spermatogenic efficiency, phylogeny, and mating system, Biol. Reprod., 69, 582591, 2003.

Zühlke, U. and Weinbauer, G.: The common marmoset (Callithrix jacchus) as a model in toxicology, Toxicol. Pathol. Suppl., 123127, 2003. 